\title{
DEVELOPMENT OF BALANCED SCORE CARD FRAMEWORK FOR PERFORMANCE EVALUATION OF AIRLINES
}

\author{
Yandava Karun Kumar \\ Part-time Ph.D. Scholar in Department of Mechanical Engineering, \\ College of Engineering(A), Andhra University, Visakhapatnam, India \\ V.V.S. Kesava Rao \\ Professor, Department of Mechanical Engineering, \\ College of Engineering(A), Andhra University, Visakhapatnam, India
}

\begin{abstract}
The paper aims to design a multidimensional performance measurement system for airlines in order to address the complexity of balancing objectives and operational activities. The research design and methodology uses secondary data collection i.e. annual reports and business reports of airlines which are collected from the airlines own website. The paper develops and applies a customized version of balanced scorecard based on a new set of performance measures. Data on eleven performance enablers for a case study of 100 world major airlines during 2009 to 2013 is considered in the study to develop performance measurement framework of Airlines based on balanced scorecard. The Measurement of Research Construct Adopted from Wu and Laio (2014) is validated through confirmatory factor analysis using Lisrel 8.8 and developed modified set of indicators of the constructs. Further, CRITIC method is used to determine relative weights of the indicators under respective perspectives of balanced scorecard.
\end{abstract}

Keywords: Balance score card, Confirmatory analysis, critic method.

Cite this Article: Yandava Karun Kumar and V.V.S. Kesava Rao, Development of Balanced Score Card Framework for Performance Evaluation of Airlines, International Journal of Management (IJM), 10 (6), 2019, pp. 214-234.

http://iaeme.com/Home/issue/IJM?Volume $=10 \&$ Issue $=6$

\section{INTRODUCTION}

In any business environment the measurement of performance of productive system of goods or services is basic management instrument for taking of decisions and for the establishment of new actions, also serving as an aid for new strategically plans for business improvement. An efficient and accurate performance evaluation system is essential as a useful tool for the managers to control, monitor and improve the performance of the organization. Effective performance evaluation systems have the following features: Comprehensiveness, 
Comparability, Measurability and compatibility with goals. Among the advantages of performance evaluation are identification of opportunities for improvement and justification of the decisions for stakeholders.

The global airline industry provides a service to virtually every country in the world, and has played an integral role in the creation of a global economy. The airline industry itself is a major economic force, in terms of both its own operations and its impacts on related industries. Currently, airlines have suffered from high levels of competition and economic pressure, with high volatility in fuel and foreign exchange rates, shrinking fuelled by consolidation and bankruptcies, mergers, capacity cuts, bankruptcy filings, large scale losses and high debt levels. Over the last few decades, the problem of performance evaluation has attracted significant attention which led to variety of methods that seek to develop measures to assess the performance of organizations in general and airline industry in specific to deal with the performance measurement technique.

The balanced scorecard approach is makes explicit links between different performance dimensions for the evaluation of a complex system, such as airline organizations. Major issue with the BSC is the tendency towards financial measures that managers may pay insufficient attention to leading and non-financial measures causes the failure of the purpose of the Balanced Scorecard. The Balanced Scorecard has been successfully implemented across a wide number of industries and geographical locations. Organizations must foster involvement, consistency and adaptability in order to achieve measurable results from the implementation of a BSC (Deem, et al., 2010).

\section{LITERATURE SURVEY}

Gusnardi et al., (2017) aimed to analyze the dimensions of balanced scorecard as a representation of strategic planning and management system as a new style of government areas through CFA. The population and the sample used are of local government officials serving the financial statements of local government in North Sumatra as many as 361 respondents with purposive sampling methods.

Najafi, et al., (2009) identified the four perspectives including financial, customer, internal processes, and learning and growth perspectives and tries to make a balance between financial goals and the other remained perspectives.

The BSC particularly identifies the cause-and-effect relationship among leading indicators and lagging indicators (Eilat et. al., 2008).

The balanced scorecard facilitates decision making using a variety of accounting measures from different entity perspectives; traditionally, these perspectives are labeled financial, customer, internal business process, and learning and growth (Hank et al., 2015).

Balanced Scorecard measures are specifically selected to assess whether strategic objectives are being achieved (Cheng \& Humphreys, 2012; Humphreys \& Trotman, 2011; Kaplan, Petersen, \& Samuels, 2012; Libby, Malina et al., 2007).

Hasan Dinçer et al., (2017) evaluated the performance of the European airlines, using a balanced scorecard perspective and recommend European airlines to focus on these aspects in order to improve their performance.

Anirut Pipatprapa et al., (2016) combined structural equation modeling (SEM) and fuzzy analytic hierarchy process (FAHP) to identify the priority weight of factors for assessing the Environmental Performance (EP) of Taiwan's food industry. A SEM approach was used to develop and define EP factors and sub-factors using a questionnaire designed to gather data from 163 managers in the food industry. A FAHP approach was employed to prioritize the scores using a questionnaire to interview 21 experts regarding the different aspects of EP. 
Bojuwon Mustapha and Bojuwon Yusuf Bolaji (2015) measured lecturers' commitment scale using second other confirmatory factor analysis on the hypothesized model with four latent variables of commitment to student, commitment to teaching, commitment to school and commitment to the profession.

Shahid Hussain et al., (2018) applied SEM approach to identify the critical factors affecting quality in social infrastructure projects. The authors developed a quantitative approach using smart-PLS version 3.2.7.

David Staš et al., (2015) designed a conceptual framework for creating the Green Transport (GT) Balanced Scorecard (BSC) models from the viewpoint of industrial companies and supply chains using Analytic network Process (ANP).

Agnieszka Tubis and Sylwia Werbińska-Wojciechowska (2017) presented elements of the BSC corresponding to the general trends of changes and strategies currently being undertaken by the companies in the road passenger transport sector.

Frederico, Guilherme F and Cavenaghi, Vagner (2008), presented a performance management model, based in the Balanced Scorecard, through a case study of Brazilian freights railroad transportation and approaches four Balanced Scorecard's perspectives (financial, customers, processes and learning and growth).

Hamed Rahimi et al., (2017) analyzed systematic relations between key indicators of hospital performance evaluation, identifying causal relations and prioritizing indicators based on the 4 perspectives described by the balanced scorecard (BSC).

Saif Hassan Ali Alzaabi and Hassan Al-Dhaafri (2018) investigated the effects of Strategy Management (ST) and Balanced Scorecard (BSC) on Organizational Performance (OP). Based on a theoretical foundation and a wide review of the literature, the model of the research was proposed 220 Questionnaires were distributed among random selected sample of Civil Defense departments in the Northern Emirates using structured equation modeling

Vinh V. Thai (2015), investigated the concept of Port Service Quality (PSQ) and examines its influence on customer satisfaction in the port sector. Following a literature review, a conceptual model of PSQ and its influence on customer satisfaction is proposed. Confirmatory factor analysis, followed by multiple regression is conducted to confirm the PSQ construct and examine the relationship between PSQ and customer satisfaction.

\section{CONFIRMATORY FACTOR ANALYSIS OF BALANCED SCORECARD}

$\mathrm{Wu}$ and Laio (2014) proposed balanced scorecard indicators in respect of airline organizations and are presented in Table-1.

Table 1: Balanced scorecard constructs (Wu and Laio, 2014)

\begin{tabular}{|c|c|}
\hline Construct & Indicator \\
\hline Financial Perspective & Operating Revenue (OR) \\
& Return On Investment (ROI) \\
& Return On Assets (ROA) \\
& Net Income (NI) \\
\hline Customer Perspective & Revenue Passenger Kilometer (RPK) \\
& Number of Passengers (NP) \\
& Revenue Ton Kilometer (RTK) \\
\hline Internal Business Perspective & Energy (Fuel) Cost ( EC) \\
& Capital Cost (CC) \\
\hline Learning and Growth Perspective & Labor Cost (LC) \\
& Operating Expenses per employee (OE) \\
\hline
\end{tabular}




\section{METHODOLOGY}

To conduct CFA, initially items are generated that accurately reflect the proposed constructs of the subject under consideration based on a comprehensive literature review and comments from an expert panel. A questionnaire is developed for each of the construct with measurement items. Respondents (stake holders) are asked to measure their level of agreement for each of the construct items on a Likert scale (i.e. strongly disagree to agree strongly). Then CFA is implemented to assess how well the observed variables reflect the proposed constructs using some statistical softwares like AMOS, Lisrel etc. In this study, instead of primary data collection from questionnaire, secondary data collection from annual reports, financial statements etc. is made to conduct CFA.

Integrated methodology for development of framework of balanced scorecard for evaluation of is explained below. In this study, data on 11 parameters of 100 airlines are considered to develop the framework. The flow diagram of the methodology is presented in Fig.1.

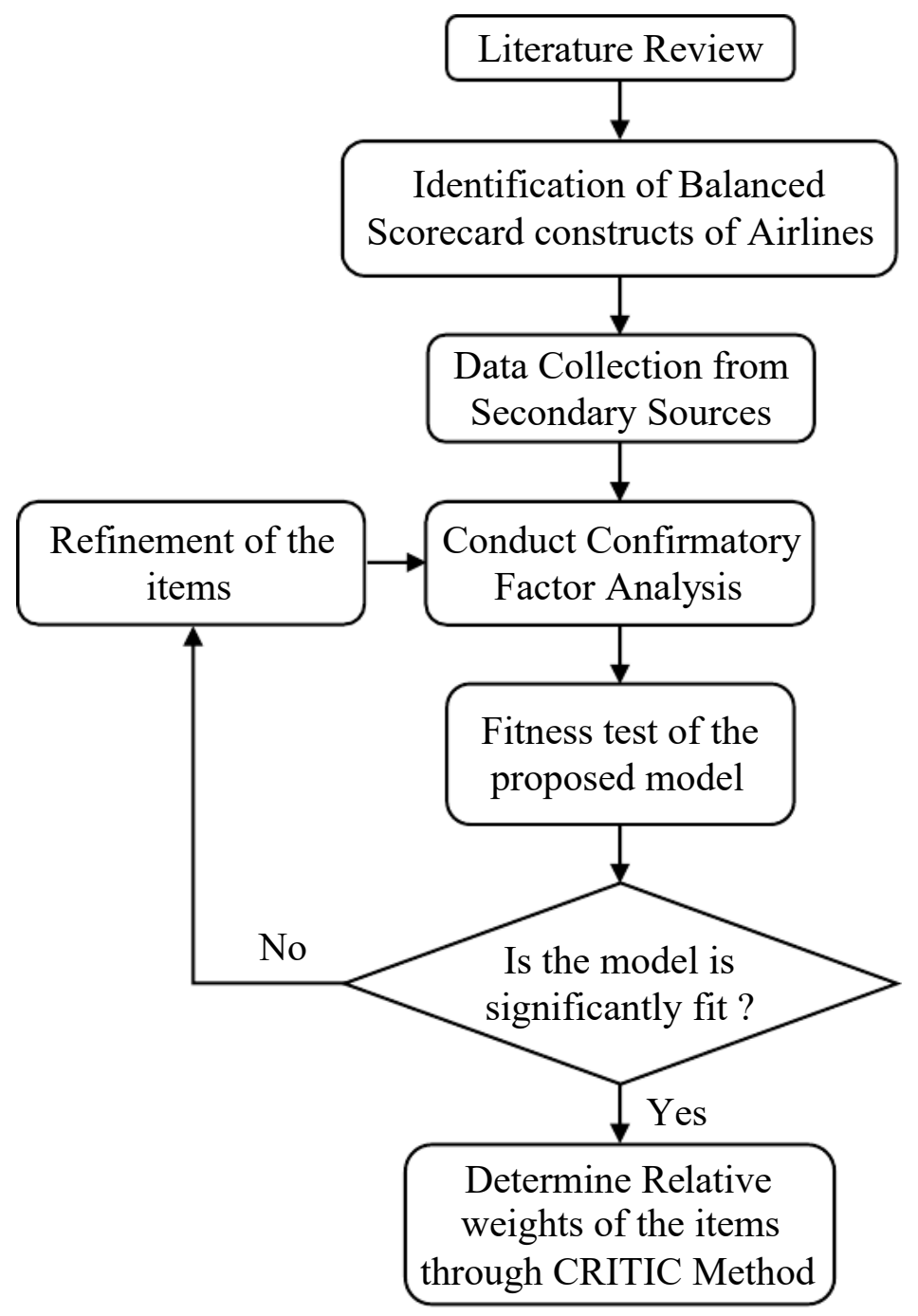

Figure 1: Flow diagram of the proposed methodology 
The stepwise methodology is discussed below.

Step-1: Consider hypothesized Model of balanced scorecard

Hypothesized measurement model of balanced scorecard performance of Airlines namely: (i) financial perspective (FP) (ii) Customer Perspective (CP) (iii) Internal Business perspective (IBP) and (iv) Learning and Growth perspective (LGP) is considered and is shown in Fig.2.

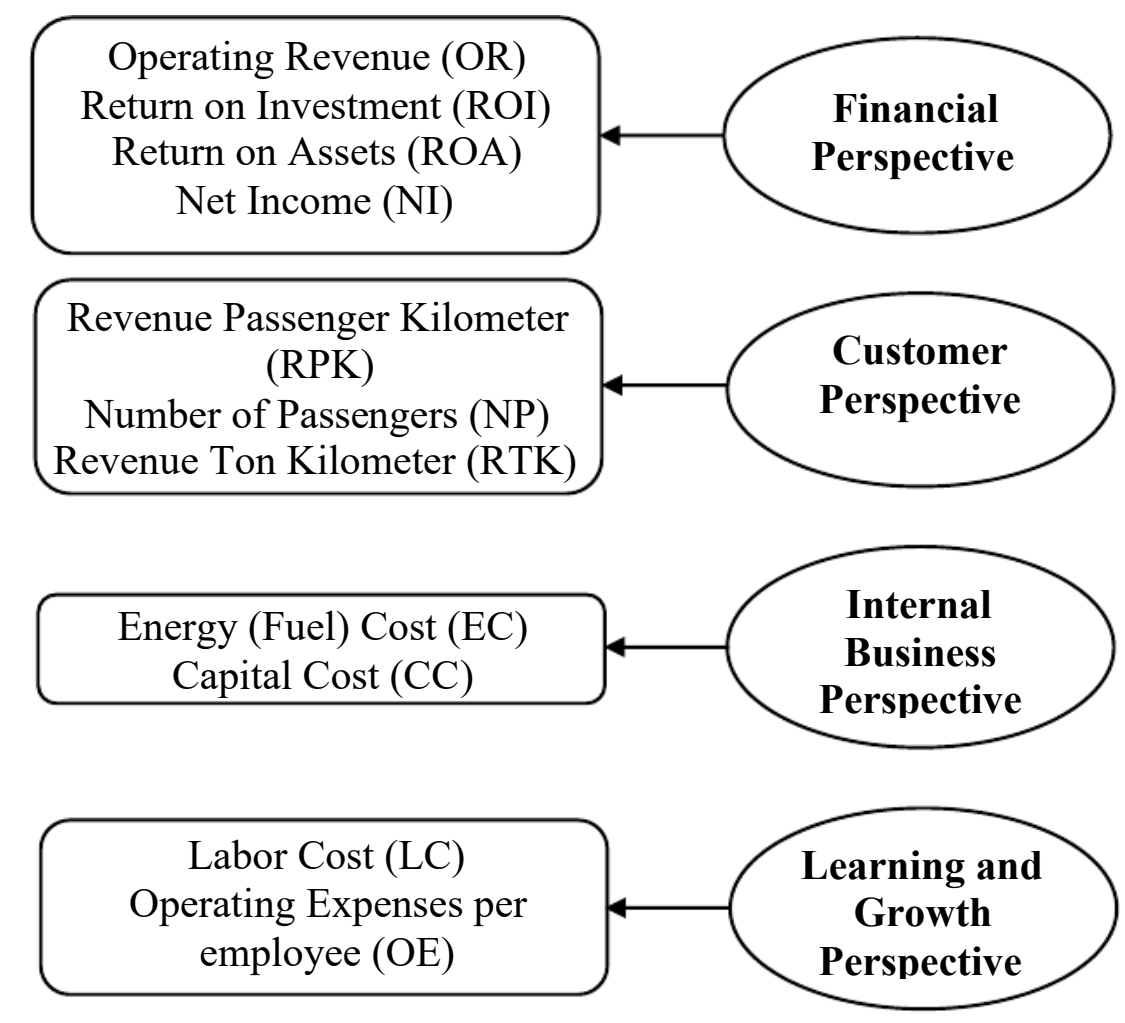

Figure 2 Hypothesized model of balanced scorecard of airlines

Step-2: Collect the data

Obtain the data on 11 items of the balanced scorecard perspectives from secondary sources of data (annual reports, financial statements).

Step-3: Formulate Correlation matrix

The proposed approach utilizes correlations among the items of the perspectives of Airlines.

Step-4: Perform Confirmatory Factor Analysis

Confirmatory factor analysis was adopted to validate the hypothesized measurement model of balanced scorecard performance of Airlines using correlation matrix.

Step-5: Determine Goodness of fit:

Goodness of fit indices are determined from the confirmatory factor analysis. The fit model is an indication of relation between the observed variables (criteria) and their latent variable.

Step-6: Determine relative weights of the observed variables

In this study, CRITIC method is proposed to determine relative weights of the observed variables (items)

Step-6.1: Obtain Data on the validated criteria

Obtain the data on the criteria of the balanced scorecard perspectives as validated in the CFA. 
Step-6.2: Normalize the data

Normalize the data using the relations as discussed in section 4.1

Step-6.3: Determine Standard deviation

Standard deviations of the criteria are determined as discussed in section 4.2

Step-6.4: Determine measure of conflict

Measures of conflict of the criteria are determined as discussed in section 4.3

Step-6.5: Determine Information content

Information content of the all the criteria are determined as discussed in section 4.4

Step-6.6: Determine relative weight

Relative weights of the criteria are determined as discussed in section 4.4

\section{CASE STUDY}

The study is developed based on an empirical study of selected 20 Airlines. The secondary data on 11 criteria during 2009-2013 in the study is obtained from annual reports of respective Airline companies. A comprehensive research methodology developed and shown in Fig.1 is implemented. CFA technique is used to test the hypothesis that a relationship exists between the observed variables (Criteria) and their underlying latent constructs. A software tool Lisrel 8.8 has been used for CFA analysis. Relative weights of the validated criteria are obtained by implementing CRITIC method.

\subsection{Data on 11 items of 20 Airlines}

Data on 11 criteria is collected from financial reports is presented in Table-2.

Table-2: Financial and traffic data of the airlines

\begin{tabular}{|c|c|c|c|c|c|c|c|c|c|c|c|c|c|}
\hline S.No & Airlines & $\begin{array}{c}\text { Yea } \\
\mathbf{r}\end{array}$ & $\begin{array}{c}\text { OR } \\
\text { (million } \\
\$ \$\end{array}$ & $\begin{array}{l}\text { ROI } \\
(\%)\end{array}$ & $\begin{array}{l}\text { ROA } \\
(\%)\end{array}$ & \begin{tabular}{|c|}
$\mathbf{N l}$ \\
$(\mathbf{m i l l i o n}$ \\
$\$ \mathbf{S})$
\end{tabular} & \begin{tabular}{|c|} 
RPK \\
(million \\
$\$$ )
\end{tabular} & \begin{tabular}{|c}
$\mathbf{N P}$ \\
(millions \\
/ year)
\end{tabular} & \begin{tabular}{|c|} 
RTK \\
(million \\
$\$$ )
\end{tabular} & $\begin{array}{c}\mathbf{E C} \\
\text { (million } \\
\$ \text { ) }\end{array}$ & $\begin{array}{c}\mathbf{C C} \\
\text { (million } \\
\$ \text { ) }\end{array}$ & \begin{tabular}{|c|}
$\mathbf{L C}$ \\
(million \\
$\mathrm{S})$
\end{tabular} & \begin{tabular}{|c|}
$\mathbf{O E}$ \\
(million \\
$\$$ \$)
\end{tabular} \\
\hline 1 & Aer Lingus & 2009 & 866.035 & 3.55 & 4.99 & 32.039 & 5400 & 6.6 & 132.098 & 148.942 & 200.611 & 125.113 & \begin{tabular}{|l}
297.013 \\
\end{tabular} \\
\hline 2 & Aer Lingus & 2010 & 1610.262 & 5.21 & 2.73 & 65.174 & 13895 & 9.346 & 216.628 & 352.632 & 202.916 & 342.945 & 137.079 \\
\hline 3 & Aer Lingus & 2011 & 1655.283 & 7.56 & 3.6 & 9.148 & 14051 & 9.513 & 240.533 & 370.975 & 126.692 & \begin{tabular}{|l|}
334.77 \\
\end{tabular} & 36.704 \\
\hline 4 & Aer Lingus & 2012 & 1790.193 & 3.21 & 1.91 & 43.814 & 14523 & 9.653 & 239.415 & 460.699 & 184.908 & \begin{tabular}{|l|}
342.754 \\
\end{tabular} & 139.212 \\
\hline 5 & Aer Lingus & 2013 & 1892.45 & 3.12 & 1.96 & 45.283 & 14807 & 9.625 & 259.498 & 474.474 & 205.277 & 368.371 & 142.976 \\
\hline 6 & Aeroflot & 2009 & 3345.9 & 3.21 & 2.15 & 85.8 & 29900 & 8.756 & 1118.5 & 725.4 & 53.1 & 538.9 & 233.5 \\
\hline 7 & Aeroflot & 2010 & 4319.3 & 7.38 & 5.6 & 253.2 & 39200 & 11.286 & 1312.2 & 943 & 159.5 & 686.5 & 330.9 \\
\hline 8 & Aeroflot & 2011 & 537 & $\begin{array}{c}10.4 \\
2\end{array}$ & 9.21 & 491.3 & 460 & 14.174 & & 147 & 244.2 & 0.1 & \\
\hline 9 & roflot & 2012 & 8138.1 & 2.38 & 2.66 & 166.3 & 74617.2 & 27.5 & 2682.3 & 2287.5 & 166.7 & \begin{tabular}{|l|}
1241.8 \\
\end{tabular} & 614.1 \\
\hline 10 & Aeroflot & 2013 & 9133.754 & 2.51 & 3.51 & 230.262 & 85300 & 31.4 & $\begin{array}{c}2959.72 \\
1\end{array}$ & 2484.003 & 276.691 & \begin{tabular}{|c|}
1423.60 \\
6
\end{tabular} & 2018.237 \\
\hline 11 & sia & 2009 & 892.194 & $\begin{array}{c}14.3 \\
3\end{array}$ & 4.44 & 144.171 & 16890 & 14.253 & 254.892 & 264.211 & 517.405 & 87.141 & 111.837 \\
\hline 12 & Air Asia & 2010 & 19 & $\begin{array}{c}38.8 \\
9\end{array}$ & 8.02 & 6 & 18499 & 16.055 & 27 & 58 & 77 & 03 & 26 \\
\hline 13 & Air Asia & 2011 & 1469.657 & $\begin{array}{c}28.8 \\
5\end{array}$ & 8.36 & 380.247 & 21037 & 17.987 & 28.346 & 575.395 & 404.158 & \begin{tabular}{|l|}
158.303 \\
\end{tabular} & \begin{tabular}{|l|}
151.778 \\
\end{tabular} \\
\hline 14 & ia & 2012 & 1601.912 & $\begin{array}{c}25.2 \\
1\end{array}$ & 6.13 & 332.625 & 22731 & 19.679 & 36.403 & 630.902 & 318.199 & \begin{tabular}{|l|}
187.946 \\
\end{tabular} & 146.169 \\
\hline 15 & Air Asia & 2013 & 1622.94 & 7.51 & 2.03 & 114.97 & 26607 & 21.853 & 44.011 & 702.346 & 421 & \begin{tabular}{|l|}
193.955 \\
\end{tabular} & \begin{tabular}{|l|}
169.007 \\
\end{tabular} \\
\hline 16 & Air China & 2009 & 7523.592 & 9.73 & 4.45 & 703.132 & 75473.77 & 41.279 & 629.895 & 2117.727 & $\begin{array}{c}1543.87 \\
3\end{array}$ & 970.205 & 1962.544 \\
\hline 17 & Air China & 2010 & \begin{tabular}{|c|}
12184.34 \\
5
\end{tabular} & $\begin{array}{c}17.1 \\
2\end{array}$ & \begin{tabular}{|l|}
7.77 \\
\end{tabular} & $\begin{array}{c}1822.14 \\
7\end{array}$ & 105695 & 60.006 & 924.58 & 3559.264 & \begin{tabular}{|c|}
1678.15 \\
5
\end{tabular} & \begin{tabular}{|c|}
1455.24 \\
3 \\
\end{tabular} & 3033.595 \\
\hline 18 & Air China & 2011 & 15220.64 & 7.72 & 4.02 & \begin{tabular}{|c|}
1092.35 \\
7
\end{tabular} & $\begin{array}{c}123489.0 \\
7\end{array}$ & 69.687 & \begin{tabular}{|c}
1165.75 \\
3
\end{tabular} & 5367.444 & $\begin{array}{c}2065.93 \\
5\end{array}$ & \begin{tabular}{|c|}
1897.76 \\
6
\end{tabular} & 3644.6 \\
\hline
\end{tabular}


Development of Balanced Score Card Framework for Performance Evaluation of Airlines

\begin{tabular}{|c|c|c|c|c|c|c|c|c|c|c|c|c|c|}
\hline S.No & Airlines & $\begin{array}{c}\text { Yea } \\
\mathbf{r} \\
\end{array}$ & \begin{tabular}{|c|} 
OR \\
(million \\
$\$)$
\end{tabular} & $\begin{array}{l}\text { ROI } \\
(\%)\end{array}$ & $\begin{array}{l}\text { ROA } \\
(\%)\end{array}$ & \begin{tabular}{|c|} 
Nl \\
$(\mathrm{million}$ \\
$\$)$
\end{tabular} & \begin{tabular}{|c|} 
RPK \\
(million \\
$\$)$
\end{tabular} & $\begin{array}{c}\text { NP } \\
\text { (millions } \\
\text { / year) }\end{array}$ & $\begin{array}{c}\text { RTK } \\
\text { (million } \\
\$)\end{array}$ & \begin{tabular}{|c} 
EC \\
(million \\
$\$)$ \\
\end{tabular} & $\begin{array}{c}\mathrm{CC} \\
\text { (million } \\
\$)\end{array}$ & \begin{tabular}{|c|} 
LC \\
(million \\
S) \\
\end{tabular} & \begin{tabular}{|c} 
OE \\
(million \\
$\$$ )
\end{tabular} \\
\hline 19 & Air China & 2012 & 15979.43 & 5.14 & 2.63 & 780.925 & \begin{tabular}{|c}
129773.3 \\
2
\end{tabular} & 72.416 & $\begin{array}{c}1109.14 \\
9\end{array}$ & 5647.52 & $\begin{array}{c}2833.97 \\
8\end{array}$ & $\begin{array}{c}2162.78 \\
6\end{array}$ & 3445.072 \\
\hline 20 & Air China & 2013 & $\begin{array}{c}15958.40 \\
9 \\
\end{array}$ & 3.82 & 1.76 & 587.58 & $\begin{array}{c}141967.9 \\
5\end{array}$ & 77.677 & \begin{tabular}{|c|}
1114.48 \\
3
\end{tabular} & 5481.255 & $\begin{array}{c}2659.26 \\
5\end{array}$ & \begin{tabular}{|c|}
2279.41 \\
7 \\
\end{tabular} & 3836.409 \\
\hline 21 & $\begin{array}{c}\text { Air } \\
\text { Newzealan } \\
\text { d } \\
\end{array}$ & 2009 & 2995.85 & 0.33 & 0.42 & 13.65 & 27112 & 12.4 & 616.85 & 109.655 & 1383.2 & \begin{tabular}{|l|}
662.35 \\
\end{tabular} & 361.4 \\
\hline 22 & $\begin{array}{c}\text { Air } \\
\text { Newzealan } \\
\text { d } \\
\end{array}$ & 2010 & 2797.809 & 1.66 & 1.78 & 56.703 & 25829 & 12.3 & 614.052 & 649.319 & $\begin{array}{c}1144.43 \\
3\end{array}$ & 674.904 & 341.601 \\
\hline 23 & $\begin{array}{c}\text { Air } \\
\text { Newzealan } \\
\text { d } \\
\end{array}$ & 2011 & 3576.984 & 1.53 & 1.65 & 66.744 & 26996 & 13.103 & 745.72 & 893.216 & $\begin{array}{c}1452.71 \\
2\end{array}$ & 852.016 & 418.592 \\
\hline 24 & $\begin{array}{c}\text { Air } \\
\text { Newzealan } \\
\mathrm{d} \\
\end{array}$ & 2012 & 3530.363 & 1.53 & 1.3 & 55.913 & 27013 & 13.122 & 746.55 & 959.963 & 1370.25 & 826.875 & 397.688 \\
\hline 25 & $\begin{array}{c}\text { Air } \\
\text { Newzealan } \\
\mathrm{d} \\
\end{array}$ & 2013 & 3602.184 & 3.07 & 3.25 & 141.966 & 27733 & 13.411 & 771.451 & 939.158 & $\begin{array}{c}1641.18 \\
6\end{array}$ & 833.853 & 433.697 \\
\hline 26 & $\begin{array}{c}\text { Allegiant } \\
\text { Air }\end{array}$ & 2009 & 557.94 & \begin{tabular}{|c|}
15.8 \\
5 \\
\end{tabular} & $\begin{array}{c}15.2 \\
8 \\
\end{array}$ & 76.331 & 4762.41 & 5.328 & 52.938 & 165 & 77.486 & 90.006 & 96.179 \\
\hline 27 & $\begin{array}{c}\text { Allegiant } \\
\text { Air }\end{array}$ & 2010 & 663.641 & \begin{tabular}{|l|}
9.9 \\
\end{tabular} & $\begin{array}{c}13.1 \\
1 \\
\end{array}$ & 65.702 & 5466.237 & 5.903 & 60.579 & 243.671 & 141.342 & 108 & 110.049 \\
\hline 28 & $\begin{array}{c}\text { Allegiant } \\
\text { Air }\end{array}$ & 2011 & 779.117 & 6.34 & 6.99 & 49.398 & 5640.577 & 6.176 & 81.228 & 330.657 & 128.52 & 119.856 & 118.856 \\
\hline 29 & $\begin{array}{c}\text { Allegiant } \\
\text { Air }\end{array}$ & 2012 & 908.719 & 9.44 & 9.82 & 78.414 & 6514.056 & 6.987 & 73.897 & 378.195 & 111.393 & 133.295 & 133.525 \\
\hline 30 & $\begin{array}{c}\text { Allegiant } \\
\text { Air }\end{array}$ & 2013 & 996.15 & \begin{tabular}{|c|}
10.1 \\
5 \\
\end{tabular} & 9.87 & 91.779 & 7129.416 & 7.241 & 72.818 & 385.558 & 141.449 & 158.627 & 145.919 \\
\hline 31 & $\begin{array}{l}\text { Cathay } \\
\text { Pacific }\end{array}$ & 2009 & 8587 & 5.78 & 6.03 & 623 & 89440 & 24.558 & 3834 & 2224 & 1309 & 1618 & 1791 \\
\hline 32 & $\begin{array}{l}\text { Cathay } \\
\text { Pacific }\end{array}$ & 2010 & 11477 & $\begin{array}{c}13.6 \\
2 \\
\end{array}$ & $\begin{array}{c}15.5 \\
5\end{array}$ & 1825 & 96588 & 26.796 & 4363 & 3625 & 1516 & 1776 & 2124 \\
\hline 33 & $\begin{array}{l}\text { Cathay } \\
\text { Pacific }\end{array}$ & 2011 & 12616 & 4.87 & 5.66 & 727 & 101536 & 27.581 & 4380 & 4984 & 1404 & 1894 & 2255 \\
\hline 34 & $\begin{array}{l}\text { Cathay } \\
\text { Pacific }\end{array}$ & 2012 & 12741 & 0.93 & 0.95 & 145 & 103837 & 28.961 & 4542 & 5188 & 1488 & 2061 & 2375 \\
\hline 35 & $\begin{array}{l}\text { Cathay } \\
\text { Pacific }\end{array}$ & 2013 & 12882 & 2.33 & 2.51 & 372 & 104571 & 29.92 & 4871 & 4889 & 1687 & 2183 & 2361 \\
\hline 36 & $\begin{array}{c}\text { Cebu } \\
\text { Pacific Air } \\
\end{array}$ & 2009 & 489.885 & \begin{tabular}{|c|}
14.3 \\
2 \\
\end{tabular} & 9.22 & 68.464 & 7056 & 8.756 & 68.651 & 154.677 & 95.085 & \begin{tabular}{|l|}
27.096 \\
\end{tabular} & 132.561 \\
\hline 37 & $\begin{array}{c}\text { Cebu } \\
\text { Pacific Air } \\
\end{array}$ & 2010 & 645.176 & \begin{tabular}{|c|}
28.1 \\
2 \\
\end{tabular} & $\begin{array}{c}13.8 \\
6 \\
\end{array}$ & 153.538 & 8860 & 10.461 & 76.151 & 217.533 & 68.139 & 43.513 & 40.904 \\
\hline 38 & $\begin{array}{c}\text { Cebu } \\
\text { Pacific Air } \\
\end{array}$ & 2011 & 784.013 & \begin{tabular}{|c|}
10.8 \\
8 \\
\end{tabular} & 7.26 & 83.735 & 10531 & 11.933 & 91.474 & 351.646 & 93.791 & \begin{tabular}{|l|}
56.845 \\
\end{tabular} & 175.631 \\
\hline 39 & $\begin{array}{c}\text { Cebu } \\
\text { Pacific Air }\end{array}$ & 2012 & 898.146 & $\begin{array}{c}49.5 \\
6 \\
\end{array}$ & 6.41 & 84.639 & 11533 & 13.255 & 104.236 & 416.128 & 100.484 & 72.084 & 204.818 \\
\hline 40 & $\begin{array}{c}\text { Cebu } \\
\text { Pacific Air } \\
\end{array}$ & 2013 & 966.117 & 1.16 & 0.76 & 12.062 & 12927 & 14.352 & 114.46 & 459.984 & 190.554 & 64.305 & 209.639 \\
\hline 41 & $\begin{array}{c}\text { China } \\
\text { Eastern } \\
\text { Airlines }\end{array}$ & 2009 & 5707.805 & 0.44 & 0.27 & 28.789 & 60942.09 & 44.043 & $\begin{array}{c}1359.70 \\
4\end{array}$ & 1794.041 & 1574.05 & 753.758 & 1131.339 \\
\hline 42 & $\begin{array}{c}\text { China } \\
\text { Eastern } \\
\text { Airlines }\end{array}$ & 2010 & $\begin{array}{c}10901.63 \\
7\end{array}$ & 7.43 & 5.11 & 780.554 & 93152.76 & 4.93 & $\begin{array}{c}2344.64 \\
9\end{array}$ & 3191.394 & $\begin{array}{c}1986.53 \\
5\end{array}$ & \begin{tabular}{|c|}
1320.65 \\
5
\end{tabular} & 1657.045 \\
\hline 43 & $\begin{array}{c}\text { China } \\
\text { Eastern } \\
\text { Airlines }\end{array}$ & 2011 & $\begin{array}{c}12744.99 \\
2\end{array}$ & 5.78 & 3.99 & 707.826 & $\begin{array}{c}100895.0 \\
6\end{array}$ & 68.725 & $\begin{array}{c}2363.61 \\
4\end{array}$ & 4520.745 & $\begin{array}{c}2065.63 \\
2\end{array}$ & \begin{tabular}{|c|}
1340.16 \\
1
\end{tabular} & 1957.676 \\
\hline 44 & $\begin{array}{c}\text { China } \\
\text { Eastern } \\
\text { Airlines }\end{array}$ & 2012 & $\begin{array}{c}13509.82 \\
5\end{array}$ & 3.32 & 2.27 & 444.912 & $\begin{array}{c}109112.6 \\
8\end{array}$ & 73.077 & $\begin{array}{c}2447.80 \\
5\end{array}$ & 4733.644 & $\begin{array}{c}2499.70 \\
1\end{array}$ & \begin{tabular}{|c|}
1594.02 \\
5
\end{tabular} & 2121.418 \\
\hline
\end{tabular}




\begin{tabular}{|c|c|c|c|c|c|c|c|c|c|c|c|c|c|}
\hline No & nes & $\begin{array}{c}\text { Yea } \\
\text { r }\end{array}$ & $\begin{array}{c}\begin{array}{c}\text { OR } \\
\text { (million }\end{array} \\
\$ \text { ) }\end{array}$ & $\begin{array}{l}\text { ROI } \\
(\%)\end{array}$ & $\begin{array}{l}\text { ROA } \\
(\%)\end{array}$ & 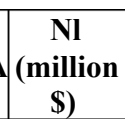 & $\begin{array}{c}\begin{array}{c}\text { RPK } \\
\text { (million }\end{array} \\
\$ \text { ) }\end{array}$ & $\begin{array}{c}\mathbf{N P} \\
\text { (millions } \\
\text { / year) }\end{array}$ & $\begin{array}{c}\text { RTK } \\
\text { (million } \\
\$)\end{array}$ & $\begin{array}{c}\text { EC } \\
\text { (million } \\
\$)\end{array}$ & $\begin{array}{c}\mathrm{CC} \\
\text { (million } \\
\$ \text { ) }\end{array}$ & \begin{tabular}{|c} 
LC \\
(million \\
$\mathbf{S})$
\end{tabular} & $\begin{array}{c}\text { OE } \\
\text { (million } \\
\$)\end{array}$ \\
\hline 45 & $\begin{array}{c}\text { China } \\
\text { Eastern } \\
\text { Airlines }\end{array}$ & 2013 & $\begin{array}{c}14343.43 \\
5\end{array}$ & 2.36 & 1.49 & 340.198 & $\begin{array}{c}120461.1 \\
3\end{array}$ & 79.094 & $\begin{array}{c}1882.38 \\
8\end{array}$ & 4986.922 & $\begin{array}{c}2396.66 \\
8\end{array}$ & $\begin{array}{c}2186.82 \\
7\end{array}$ & 2996.282 \\
\hline 46 & $\begin{array}{c}\text { Copa } \\
\text { Holdings }\end{array}$ & 2009 & 1256.076 & $\begin{array}{c}24.7 \\
1\end{array}$ & $\begin{array}{c}11.5 \\
3\end{array}$ & 249.087 & 4597.265 & 7.182 & 180.87 & 300.816 & 168.703 & 157.879 & 199.555 \\
\hline 47 & $\begin{array}{c}\text { Copa } \\
\text { Holdings }\end{array}$ & 2010 & 1414.806 & $\begin{array}{c}20.5 \\
4\end{array}$ & 9.43 & 241.057 & 5230.578 & .998 & 5.947 & 354.427 & 28.535 & 178.845 & 15.995 \\
\hline 48 & $\begin{array}{c}\text { Copa } \\
\text { Holdings }\end{array}$ & 2011 & 30.921 & $\begin{array}{c}20.4 \\
2\end{array}$ & $\begin{array}{c}20.4 \\
2\end{array}$ & 310.425 & 6 & 23 & 31.694 & 547.221 & 285.059 & 213.094 & 43.428 \\
\hline 49 & $\begin{array}{c}\text { Copa } \\
\text { Holdings }\end{array}$ & 2012 & 388 & 17.3 & \begin{tabular}{|c|}
16.9 \\
8 \\
\end{tabular} & 76 & 9 & 14 & 93 & 25.763 & 689 & 247.405 & 97.704 \\
\hline 50 & $\begin{array}{r}\mathrm{Co} \\
\text { Hold } \\
\end{array}$ & 2013 & 2608.332 & $\begin{array}{c}19.8 \\
3\end{array}$ & 19.6 & 427.471 & 9032.318 & 1.345 & 43.597 & 783.092 & 413.983 & 276.156 & 38.385 \\
\hline 51 & $\begin{array}{c}\text { Easy Jet } \\
\text { PLC }\end{array}$ & 2009 & 4159.278 & 2.63 & 1.94 & 111.047 & $5 \mathrm{C566}$ & 5.164 & 252.04 & 1258.95 & 110.735 & 547.438 & 053.437 \\
\hline 52 & $\begin{array}{c}\text { Easy Jet } \\
\text { PLC }\end{array}$ & 2010 & 1.724 & 4.09 & 3.03 & 187.339 & 6128 & 3.754 & 73.054 & $\begin{array}{c}11326.79 \\
8\end{array}$ & 292.205 & 583.792 & 2303.044 \\
\hline 53 & $\begin{array}{c}\text { Easy Jet } \\
\text { PLC } \\
\end{array}$ & 2011 & 42 & 9 & 04 & 1 & 61347 & 54.509 & 03 & 1470.426 & 55 & 58 & 09 \\
\hline 54 & $\begin{array}{l}\text { Easy Jet } \\
\text { PLC }\end{array}$ & 2012 & 06.711 & 6.97 & 5.94 & 404.051 & 5227 & 58.4 & 21.656 & 820.605 & 437.325 & 777.996 & 2438.564 \\
\hline 55 & $\begin{array}{c}\text { Easy Jet } \\
\text { PLC } \\
\end{array}$ & 2013 & 57.806 & $\begin{array}{c}10.0 \\
9\end{array}$ & 9.02 & 622.313 & 7573 & 60.758 & 331.483 & 1848.174 & 489.407 & 841.216 & 2656.555 \\
\hline 56 & $\begin{array}{r}\text { Emi } \\
\mathrm{Gr}\end{array}$ & 2009 & $\begin{array}{c}779.86 \\
3 \\
\end{array}$ & 1.64 & 1.91 & 267.277 & 1 & 1 & \begin{tabular}{|c|}
6036.41 \\
1
\end{tabular} & 3932.339 & \begin{tabular}{|c|}
2551.67 \\
7
\end{tabular} & $\begin{array}{c}1595.75 \\
1\end{array}$ & 2.734 \\
\hline 57 & $\begin{array}{r}\text { Emi } \\
\mathrm{Gr}\end{array}$ & 2010 & \begin{tabular}{|c|}
11831.11 \\
5 \\
\end{tabular} & 6.04 & 5.93 & 12 & 13 & & \begin{tabular}{|c|}
6276.98 \\
4 \\
\end{tabular} & 88 & 2611.53 & \begin{tabular}{|c|}
1727.49 \\
8 \\
\end{tabular} & 935 \\
\hline 58 & $\begin{array}{r}\mathrm{Em} \\
\mathrm{Gr}\end{array}$ & 2011 & \begin{tabular}{|c|}
14764.77 \\
4 \\
\end{tabular} & 7.53 & 7.62 & \begin{tabular}{|c|}
1481.89 \\
5
\end{tabular} & 34 & 22 & $\begin{array}{c}7325.34 \\
9 \\
\end{array}$ & .364 & \begin{tabular}{|c|}
3094.47 \\
4
\end{tabular} & \begin{tabular}{|c|}
2073.23 \\
8 \\
\end{tabular} & 2611.762 \\
\hline 59 & $\begin{array}{r}\text { Emi } \\
\mathrm{Gr}\end{array}$ & 2012 & \begin{tabular}{|c|}
16957.97 \\
1 \\
\end{tabular} & 2.04 & 2.16 & 493.599 & 160446 & 3.981 & \begin{tabular}{|c|}
8785.68 \\
1 \\
\end{tabular} & 6613.628 & \begin{tabular}{|c|}
3704.30 \\
7 \\
\end{tabular} & $\begin{array}{c}2160.61 \\
9 \\
\end{array}$ & 2889.446 \\
\hline 60 & $\mathrm{En}$ & 2013 & \begin{tabular}{|c|}
21109.65 \\
1
\end{tabular} & 2.59 & 2.36 & 655.593 & 18 & 1 & $\begin{array}{c}9869.28 \\
4\end{array}$ & 7583.694 & $\begin{array}{c}4108.88 \\
9\end{array}$ & 245.82 & 475.077 \\
\hline 61 & $\mathrm{E}$ & 2009 & 1107.521 & $\begin{array}{c}10.4 \\
4\end{array}$ & $\begin{array}{c}12.0 \\
4\end{array}$ & 1 & 9389 & 2.01 & 277.666 & 75 & 187.059 & 65 & 2 \\
\hline 62 & & 2010 & 67.027 & 9.17 & $\begin{array}{c}10.2 \\
1 \\
\end{array}$ & 122.498 & 705 & 3.15 & .856 & 793 & .006 & 1.697 & 1.894 \\
\hline 63 & & 2011 & 3.349 & 4.33 & 3.93 & 15 & & & 55 & & 65 & & 72.704 \\
\hline 64 & & 2012 & 73.053 & 2 & 2.01 & 40.666 & & & & & & & 98.353 \\
\hline 65 & & 2013 & 2098.64 & 5.84 & 3.86 & 111.969 & & & 69 & & & 228.463 & 83.446 \\
\hline 66 & $\begin{array}{r}\mathrm{Ga} \\
\text { Indo }\end{array}$ & 2009 & 1714,944 & 6 & 6.88 & 97.807 & 18000 & 11.1 & 264.091 & 478.526 & 413.353 & 159.468 & 314,397 \\
\hline 67 & $\begin{array}{r}\mathrm{Ga} \\
\mathrm{Indo}\end{array}$ & 2010 & 9.925 & 2.68 & $\begin{array}{c}3.77 \\
1\end{array}$ & .738 & & 2.7 & .805 & .16 & 567 & 2.23 & .598 \\
\hline 68 & $\begin{array}{r}\mathrm{Ga} \\
\text { Indo }\end{array}$ & 2011 & 96.328 & 2.12 & 3.96 & 26 & .7 & 4 & 54.507 & 1137.745 & 576.795 & 238.607 & .448 \\
\hline 69 & $\begin{array}{r}\mathrm{G} \\
\text { Ind }\end{array}$ & 2012 & 72.469 & 3.39 & 4.33 & 110.843 & 342.1 & 15 & 7.522 & 255.127 & 654.428 & 144.598 & 18.907 \\
\hline 70 & $\begin{array}{c}\text { Garuda } \\
\text { Indonesia }\end{array}$ & 2013 & 3759.45 & 0,32 & 0.45 & 13.583 & 1950 & 4.965 & 17.876 & 1420.139 & 843.818 & 699.821 & 706.183 \\
\hline 71 & & 2009 & 3292 & 39 & 0.93 & 6 & $\begin{array}{c}5131.13 \\
7\end{array}$ & t5 & 149 & & 57 & 776 & 783 \\
\hline 72 & $\begin{array}{l}\text { JetBlue } \\
\text { Airways }\end{array}$ & 2010 & 3779 & 2.63 & 1.47 & 9 & \begin{tabular}{|c|}
17575.51 \\
3 \\
\end{tabular} & 24,254 & 172 & 1115 & 58 & 891 & 922 \\
\hline 73 & & 2011 & 4504 & 1.95 & 1.22 & 8( & $\begin{array}{c}19078.93 \\
1\end{array}$ & 6.37 & 227 & 1664 & 60 & 94 & 976 \\
\hline 74 & JetBlue & 2012 & 4982 & 2.64 & 1.81 & 128 & 20859.54 & 8.956 & 338 & 1806 & 636 & 1044 & 1030 \\
\hline 75 & $\begin{array}{l}\text { JetBlue } \\
\text { Airways }\end{array}$ & 2013 & 5441 & .19 & 2.29 & 1 & \begin{tabular}{|c|}
22272.21 \\
9 \\
\end{tabular} & 63 & 32 & 99 & 67 & 1135 & 1129 \\
\hline 76 & Norwegian & 2009 & 1161.242 & 3.73 & 4.85 & & 10602 & 10.8 & 219.642 & 226.136 & 307.513 & 209.219 & 75.433 \\
\hline 77 & orwegian & 2010 & 1422.36 & 88 & 2.3 & 25.148 & 13774 & 13 & 258.193 & 346.22 & 381.586 & 255.363 & 94.898 \\
\hline
\end{tabular}


Development of Balanced Score Card Framework for Performance Evaluation of Airlines

\begin{tabular}{|c|c|c|c|c|c|c|c|c|c|c|c|c|c|}
\hline S.No & Airlines & $\begin{array}{c}\text { Yea } \\
\mathbf{r}\end{array}$ & $\begin{array}{c}\text { OR } \\
\text { (million } \\
\$ \text { ) }\end{array}$ & $\begin{array}{l}\text { ROI } \\
(\%)\end{array}$ & $\begin{array}{l}\text { ROA } \\
(\%)\end{array}$ & \begin{tabular}{|c|}
$\mathbf{N l}$ \\
(million \\
$\$$ \$)
\end{tabular} & \begin{tabular}{|c|} 
RPK \\
(million \\
$\$$ )
\end{tabular} & \begin{tabular}{|c|}
$\mathbf{N P}$ \\
(millions \\
/ year)
\end{tabular} & \begin{tabular}{|c|} 
RTK \\
(million \\
$\$$ )
\end{tabular} & $\begin{array}{c}\mathbf{E C} \\
\text { (million } \\
\$ \text { ) }\end{array}$ & $\begin{array}{c}\mathbf{C C} \\
\text { (million } \\
\$)\end{array}$ & \begin{tabular}{|c|}
$\mathbf{L C}$ \\
(million \\
$\mathrm{S})$
\end{tabular} & \begin{tabular}{|c|} 
OE \\
(million \\
$\$)$
\end{tabular} \\
\hline 78 & Norwegian & 2011 & 1878.824 & 1.19 & 1.36 & 21.786 & 17421 & 15.7 & 302.158 & 551.858 & 534,875 & 330.042 & 114,276 \\
\hline 79 & Norwegian & 2012 & 2209.266 & 3.94 & 3.83 & 78.455 & 20353 & 17.7 & 321.262 & 642.645 & 537.416 & 357.708 & 130.21 \\
\hline 80 & Norwegian & 2013 & 2649.832 & 2.11 & 2.06 & 54.255 & 26881 & 20.7 & 385.61 & 800.597 & \begin{tabular}{|l|l|}
797.935 \\
\end{tabular} & \begin{tabular}{|l|}
424.002 \\
\end{tabular} & 158.024 \\
\hline 81 & \begin{tabular}{|l|} 
Singapore \\
Airlines
\end{tabular} & 2009 & 8973.514 & 8.8 & 4,63 & 788.599 & 16604.88 & 18.293 & 915.815 & 4406.749 & $\begin{array}{c}1904.65 \\
8 \\
\end{array}$ & 127.009 & 1607.111 \\
\hline 82 & $\begin{array}{l}\text { Singapore } \\
\text { Airlines }\end{array}$ & 2010 & 7441.988 & 2.61 & 1.24 & 205.031 & 82882.5 & 16.48 & 81.433 & 076.926 & $\begin{array}{c}1908.28 \\
9\end{array}$ & 2.528 & 779.475 \\
\hline 83 & $\begin{array}{l}\text { Singapore } \\
\text { Airlines }\end{array}$ & 2011 & 9338.751 & 9.69 & 4.68 & 913.899 & 84801.3 & 16.647 & $\begin{array}{c}1048.74 \\
1\end{array}$ & 3639.767 & $\begin{array}{c}2518.63 \\
3\end{array}$ & 06.362 & 18.723 \\
\hline 84 & $\begin{array}{l}\text { Sing } \\
\text { Air }\end{array}$ & 2012 & 47.382 & 3.15 & 1.8 & 317.154 & 7824 & 17.155 & 1131.86 & 463 & \begin{tabular}{|c|}
1976.13 \\
8 \\
\end{tabular} & 39 & 2213.444 \\
\hline 85 & $\begin{array}{l}\text { Sing } \\
\text { Air }\end{array}$ & 2013 & 68 & 3.46 & 1.97 & 3 & 93765.6 & 21 & $\begin{array}{c}1255.34 \\
8\end{array}$ & 69 & $\begin{array}{c}1976.13 \\
8\end{array}$ & .453 & 269 \\
\hline 86 & $\begin{array}{l}\text { Sout } \\
\text { Airlin }\end{array}$ & 2009 & 10350 & 0.97 & \begin{tabular}{|l|}
0.69 \\
\end{tabular} & 99 & $\begin{array}{c}46275.14 \\
6 \\
\end{array}$ & 6.31 & 719 & 3044 & 965 & 3468 & 2055 \\
\hline 87 & $\begin{array}{l}\text { South } \\
\text { Airlin }\end{array}$ & 2010 & 12104 & 3.94 & 2.97 & 459 & $\begin{array}{c}48506.50 \\
5\end{array}$ & 88.191 & 751 & 3620 & 1345 & 3704 & 2225 \\
\hline 88 & $\begin{array}{r}\text { Sou } \\
\text { Airli }\end{array}$ & 20 & 1 & 5 & 0 & 178 & $\begin{array}{c}60647.93 \\
7\end{array}$ & 103.974 & 955 & 5644 & 1672 & 4371 & 5 \\
\hline 89 & $\begin{array}{l}\text { Sou } \\
\text { Airli }\end{array}$ & 2012 & 17088 & 2.53 & 2.26 & 421 & \begin{tabular}{|c|}
63937.21 \\
5 \\
\end{tabular} & 347 & 1132 & 6120 & 1584 & 4749 & 3082 \\
\hline 90 & $\begin{array}{r}\text { Sout } \\
\text { Airlir }\end{array}$ & 2013 & 17699 & 4.45 & 3.9 & 754 & $\begin{array}{c}64852.83 \\
8\end{array}$ & 108.076 & 1080 & 5763 & 1838 & 5035 & 3229 \\
\hline 91 & $\mathrm{Tu}$ & 2009 & 4557.787 & 6.39 & 0.07 & 362.165 & 40130 & 25.1 & \begin{tabular}{|c|}
2215.18 \\
8 \\
\end{tabular} & 987.259 & 865.272 & 913.47 & 691.376 \\
\hline 92 & Tu & 2010 & 5588.998 & 2.77 & 2.69 & 190.072 & 50 & 9.119 & 2489.34 & 1434.938 & 916.438 & \begin{tabular}{|c|}
1170.56 \\
6 \\
\end{tabular} & 860.47 \\
\hline 93 & & 2011 & 1.518 & 0.13 & 0.12 & 022 & 3 & 9 & \begin{tabular}{|c|}
2895.82 \\
2 \\
\end{tabular} & 139 & $\begin{array}{c}1154.09 \\
9\end{array}$ & \begin{tabular}{|c|}
1331.43 \\
1 \\
\end{tabular} & 82.116 \\
\hline 94 & $\begin{array}{c}\text { Turkish } \\
\text { Airlines }\end{array}$ & 2012 & 8198.595 & 6.64 & 6.15 & 641.865 & 74410 & 39.045 & \begin{tabular}{|c|}
2657.44 \\
1
\end{tabular} & 2867.251 & $\begin{array}{c}1017.54 \\
9\end{array}$ & \begin{tabular}{|l|}
1371.7 \\
\end{tabular} & \begin{tabular}{|c|}
17467.90 \\
9 \\
\end{tabular} \\
\hline 95 & $\begin{array}{c}\text { Turkish } \\
\text { Airlines } \\
\end{array}$ & 2013 & 9855.085 & 2.65 & 2.69 & 358.322 & 1997 & 8.268 & \begin{tabular}{|c|}
4074.99 \\
8 \\
\end{tabular} & 3451.314 & \begin{tabular}{|c|}
1605.45 \\
3 \\
\end{tabular} & \begin{tabular}{|c|}
1614,89 \\
7 \\
\end{tabular} & 2799.297 \\
\hline 96 & WestJet & 2009 & 1998.47 & 2.99 & 2.81 & 86.013 & $\begin{array}{c}22260.13 \\
1\end{array}$ & 4.039 & 182.513 & 499.871 & 313.321 & 409.918 & 1474.315 \\
\hline 97 & WestJet & 2010 & 2531.953 & 2.44 & 2.53 & 87.525 & $\begin{array}{c}25121.51 \\
3\end{array}$ & 15.174 & 17.986 & 654.62 & 374.526 & 514.572 & 1823.575 \\
\hline 98 & t & 2011 & 3104.689 & 3.51 & 4,28 & 150,307 & \begin{tabular}{|c|}
27177.52 \\
4
\end{tabular} & 6.041 & 88.822 & 925.762 & 453.008 & \begin{tabular}{|l|}
582.469 \\
\end{tabular} & 2036.999 \\
\hline 99 & WestJet & 2012 & 3427.419 & 5.24 & 6.47 & 242.393 & \begin{tabular}{|c|}
29384.45 \\
1 \\
\end{tabular} & 17.423 & 310.818 & 992.79 & 492.142 & 663.758 & 2163.678 \\
\hline 100 & WestJet & 2013 & 3555.241 & 5.45 & 6.49 & 260.874 & $\begin{array}{c}31522.19 \\
7\end{array}$ & 18.691 & 335.996 & 1009.091 & 492.459 & 700.278 & 2250.874 \\
\hline
\end{tabular}

\subsection{Financial Perspective}

The mean and standard deviation values of items under financial perspective are presented in Table-3.

Table-3: Data on criteria of financial perspective

\begin{tabular}{|c|l|c|c|c|c|}
\hline S. No. & \multicolumn{1}{|c|}{ Items } & Min & Max & Average & Std. Dev \\
\hline 1 & Operating Revenue (OR) & 489.885 & 21109.651 & 5879.351 & 5151.907 \\
\hline 2 & Return On Investment (ROI) & 0.13 & 49.56 & 7.2129 & 8.185063 \\
\hline 3 & Return On Assets (ROA) & 0.07 & 20.42 & 4.88641 & 4.239464 \\
\hline 4 & Net Income (NI) & 9.148 & 1825 & 303.6458 & 353.0988 \\
\hline
\end{tabular}




\subsection{Customer Perspective}

The mean and standard deviation values of items under customer perspective are presented in Table-4.

Table-4: Data on criteria of customer perspective

\begin{tabular}{|c|l|c|c|c|c|}
\hline S.No. & \multicolumn{1}{|c|}{ Items } & Min & Max & Average & Std. Dev \\
\hline 1 & Revenue Passenger Kilometer (RPK) & 4597.265 & 188618 & 47475.15 & 41995.35 \\
\hline 2 & Number of Passengers (NP) & 2.81 & 109.347 & 27.94311 & 24.80713 \\
\hline 3 & Revenue Ton Kilometer (RTK) & 28.346 & 9869.284 & 1261.903 & 1884.075 \\
\hline
\end{tabular}

\subsection{Internal Business Perspective}

The mean and standard deviation values of items under internal business perspective are presented in Table-5.

Table-5: Internal business perspective

\begin{tabular}{|c|l|c|c|c|c|}
\hline S.No. & \multicolumn{1}{|c|}{ Items } & Min & Max & Average & Std. Dev \\
\hline 1 & Energy (Fuel) Cost ( EC) & 109.655 & 11326.798 & 2062.671 & 2089.693 \\
\hline 2 & Capital Cost (CC) & 53.1 & 4108.889 & 948.9649 & 898.2091 \\
\hline
\end{tabular}

\subsection{Learning and Growth Perspective}

The mean and standard deviation values of items under learning and growth perspective are presented in Table- 6 .

Table-6: Data on criteria of learning and growth perspective

\begin{tabular}{|c|l|c|c|c|c|}
\hline S.No. & \multicolumn{1}{|c|}{ Items } & Min & Max & Average & Std. Dev \\
\hline 1 & Labor Cost (LC) & 27.096 & 5035 & 890.7852 & 1014.711 \\
\hline 2 & Operating Expenses per employee (OE) & 75.433 & 17467.909 & 1368.594 & 1960.341 \\
\hline
\end{tabular}

\section{RESULTS AND DISCUSSION}

To test the relationship between observed criteria and their constructs of balanced scorecard of Airlines, confirmatory factor analysis is adopted for the proposed conceptual model.

\subsection{Confirmatory Factor Analysis Model-I}

The proposed model is based on four main constructs of balanced scorecard namely: (i) Financial Perspective (FP) (ii) Customer Perspective (CP) (iii) Internal Business perspective (IBP) and learning and Growth Perspective (LGP)

Based on the literature review, this research suggests the following hypotheses. The detailed discussion of hypotheses development follows.

H1: $\quad$ Operating Revenue, Return on Investment, Return on Assets and Net Income are the measurement items of financial perspective.

H2: Revenue Passenger Kilometer, Number of Passengers and Revenue Ton Kilometer are the measurement items of Customer perspective.

H3: $\quad$ Energy Cost and Capital Cost are the measurement items of Internal Business Process perspective.

H4: Labour Coat and Operating Expenses per employee are the measurement items of Learning and Growth perspective

\subsubsection{Correlation Analysis}


In this study, correlation between the eleven items four perspectives is determined using Lisrel 8.8 to input the same for confirmatory factor analysis. The Correlation matrix so obtained is presented in Table-7.

Table-7: Correlation matrix

\begin{tabular}{|c|c|c|c|c|c|c|c|c|c|c|c|}
\hline Items of the Perspectives & OR & ROI & ROA & N1 & RPK & NP & RTK & EC & CC & LC & OE \\
\hline OR & 1 & & & & & & & & & & \\
\hline ROI & -0.29 & 1 & & & & & & & & & \\
\hline ROA & -0.28 & 0.64 & 1 & & & & & & & & \\
\hline N1 & 0.63 & 0.16 & 0.24 & 1 & & & & & & & \\
\hline RPK & 0.91 & -0.25 & -0.26 & 0.65 & 1 & & & & & & \\
\hline NP & 0.76 & -0.22 & -0.29 & 0.41 & 0.57 & 1 & & & & & \\
\hline RTK & 0.69 & -0.22 & -0.14 & 0.47 & 0.79 & 0.2 & 1 & & & & \\
\hline EC & 0.86 & -0.26 & -0.27 & 0.51 & 0.8 & 0.67 & 0.57 & 1 & & & \\
\hline cc & 0.86 & -0.28 & -0.31 & 0.57 & 0.87 & 0.51 & 0.72 & 0.74 & 1 & & \\
\hline LC & 0.77 & -0.29 & -0.27 & 0.38 & 0.53 & 0.85 & 0.37 & 0.61 & 0.5 & 1 & \\
\hline OE & 0.53 & -0.16 & -0.12 & 0.42 & 0.52 & 0.46 & 0.35 & 0.49 & 0.41 & 0.42 & 1 \\
\hline
\end{tabular}

To provide evidence for construct validity of latent variables (FP, CR, IBP and LGP) CFA model is assessed. Only valuable indicators of each latent variable would be maintained in model by utilizing factor loading.

\subsubsection{Analysis of Reliability and Validity}

The study tested the measurement properties of the constructs (latent variables) through confirmatory factor analysis using correlation matrix. CFA was used to evaluate how well the measurement items reflect latent variables in the hypothesized structure.

Composite Reliability (CR) of latent variables and Average Variance Extracted (AVE) of Squared Multiple Correlation (SMC), is presented in Table-8.

Table-8: Reliability and validity analytical results of container terminal supply chain performance measurement model

\begin{tabular}{|c|c|c|c|c|}
\hline $\begin{array}{l}\text { Perspectives } \\
\text { (Constructs) }\end{array}$ & Criteria & $\begin{array}{c}\text { Standardized } \\
\text { Factor Loadings }\end{array}$ & $\begin{array}{c}\text { Composite } \\
\text { Reliability (CR) } \\
\end{array}$ & AVE \\
\hline \multirow{4}{*}{ 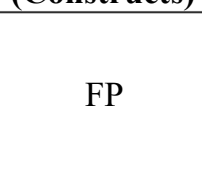 } & OR & 0.98 & \multirow{4}{*}{0.2824} & \multirow{4}{*}{0.3865} \\
\hline & ROI & -0.31 & & \\
\hline & ROA & -0.31 & & \\
\hline & NI & 0.62 & & \\
\hline \multirow{3}{*}{$\mathrm{CP}$} & RPK & 1.00 & \multirow{3}{*}{0.8437} & \multirow{3}{*}{0.6544} \\
\hline & NP & 0.57 & & \\
\hline & RTK & 0.79 & & \\
\hline \multirow{2}{*}{ BP } & $\mathrm{EC}$ & 0.84 & \multirow{2}{*}{0.8480} & \multirow{2}{*}{0.7363} \\
\hline & $\mathrm{CC}$ & 0.88 & & \\
\hline \multirow{2}{*}{ LG } & LC & 0.91 & \multirow{2}{*}{0.6593} & \multirow{2}{*}{0.5173} \\
\hline & $\mathrm{OE}$ & 0.46 & & \\
\hline
\end{tabular}

Convergent validity was verified for each factor loading. Factor loadings of ROI and ROA are poor. It indicates that these measurement items are not correlated its assumed theoretical construct.

Composite reliability of for each of the constructs of FP, CP, BP and LG are $0.2824,0.8437$, 0.8480 , and 0.6593 . Hence, the overall reliability of a set of items loaded on the FP construct is less than threshold value of 0.5 indicates the poor overall reliability of a set of items loaded on the construct. 
The average variance extracted (AVE) for each of the constructs of FP, CP, BP and LG are $0.3865,0.6544,0.7363$ and 0.5173 respectively. AVE value of FP is less than threshold value of 0.5 indicates poor convergent validity. Convergent validity is the degree of confidence we have that a latent variable is well measured by its indicators.

\subsubsection{Path Diagram}

The path diagram of the model is shown in Fig.3

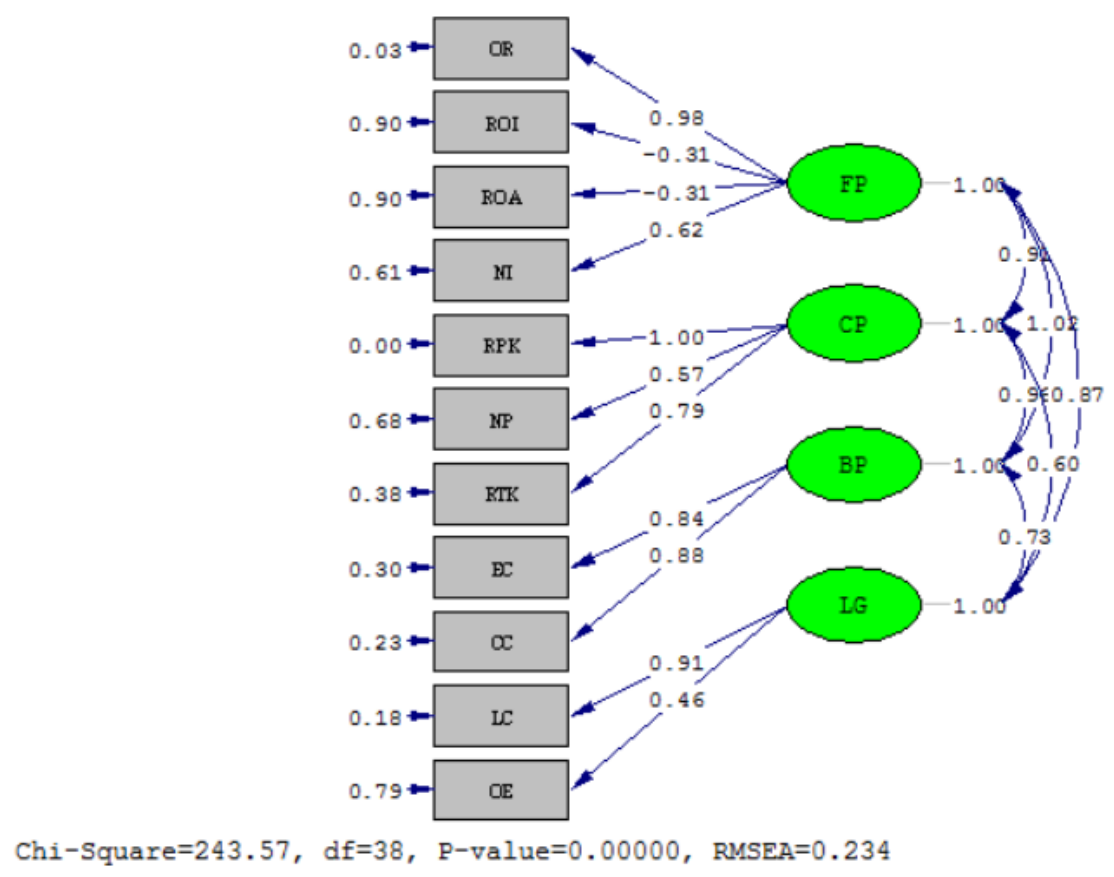

Figure 3: Path diagram

\subsubsection{Model Fit Indices}

The model fitting process involves determining the goodness-of fit between the hypothesized model and the sample data. Fit indices of the conceptual model obtained through CFA are shown in the Table-9.

Table-9: Fit indices of structure model

\begin{tabular}{|c|c|c|c|}
\hline Indicators & Propriety Indicators & Research Findings & Remarks \\
\hline \multirow{4}{*}{ Absolute Propriety Indicators } & $\chi^{2} / \mathrm{d} . f$ & 6.41 & Lack of fit \\
\cline { 2 - 4 } & GFI & 0.69 & Lack of fit \\
\cline { 2 - 4 } & AGFI & 0.46 & Lack of fit \\
\cline { 2 - 4 } & SRMR & 0.15 & Poor fit \\
\cline { 2 - 4 } & RMSEA & 0.23 & Lack of fit \\
\hline \multirow{3}{*}{ Relative Propriety Indicators } & NNFI & 0.73 & Lack of fit \\
\cline { 2 - 4 } & NFI & 0.80 & Lack of fit \\
\cline { 2 - 4 } & CFI & 0.81 & Lack of fit \\
\hline
\end{tabular}

The Chi square goodness of fit metric is obtained as 243.57. The goodness-of-fit index (GFI) of was the very first standardized fit index GFI $=0.69$ indicates poor model fit (GFI > .90). Also, AGFI, NNFI, NFI and CFI are obtained as $0.46,0.73,0.80$ and 0.81 respectively. It indicates overall poor fit of the model with the criteria under the respective construct.

In the study, RMSEA and SRMR are obtained as 0.23 and 0.15 respectively indicates the poor model fit since higher values are obtained. Overall measurement model fitness of 6.41 is 
obtained. It indicates that the overall measurement model fitness is poor since $\chi^{2} /$ d.f value is more than 3.0.

Hence, the confirmatory factor analyses with 4 constructs having 11 items is not valid measurement model of balanced scorecard of Airlines and proceed for further analysis by removing ROI and ROA factors of financial perspective.

\subsection{Confirmatory Factor Analysis Model-II}

The proposed model is based on four main constructs of balanced scorecard namely: (i) Financial Perspective (FP) (ii) Customer Perspective (CP) (iii) Internal Business perspective (IBP) and learning and Growth Perspective (LGP)

Based on the literature review, this research suggests the following hypotheses. The detailed discussion of hypotheses development follows.

H1: Operating Revenue and Net Income are the measurement items of financial perspective.

H2: Revenue Passenger Kilometer, Number of Passengers and Revenue Ton Kilometer are the measurement items of Customer perspective.

H3: $\quad$ Energy Cost and Capital Cost are the measurement items of Internal Business Process perspective.

H4: Labour Coat and Operating Expenses per employee are the measurement items of Learning and Growth perspective

\subsubsection{Correlation Analysis}

In this study, correlation between nine criteria under four perspectives is determined using Lisrel 8.8 to input the same for confirmatory factor analysis. The Correlation matrix so obtained is presented in Table-10.

Table-10: Correlation matrix

\begin{tabular}{|c|c|c|c|c|c|c|c|c|}
\hline Criteria & OR & NI & RPK & NP & RTK & EC & CC & LC \\
\hline OR & 1 & & & & & & & \\
\hline NI & 0.635 & 1 & & & & & & \\
\hline RPK & 0.908 & 0.652 & 1 & & & & & \\
\hline NP & 0.760 & 0.410 & 0.573 & 1 & & & & \\
\hline RTK & 0.690 & 0.467 & 0.792 & 0.202 & 1 & & & \\
\hline EC & 0.865 & 0.509 & 0.797 & 0.669 & 0.574 & 1 & & \\
\hline CC & 0.864 & 0.574 & 0.866 & 0.508 & 0.723 & 0.737 & 1 & \\
\hline LC & 0.765 & 0.382 & 0.526 & 0.846 & 0.369 & 0.613 & 0.503 & 1 \\
\hline
\end{tabular}

To provide evidence for construct validity of latent variables (FP, CR, IBP and LGP) CFA model is assessed. Only valuable indicators of each latent variable would be maintained in model by utilizing factor loading.

\subsubsection{Analysis of Reliability and Validity}

The study tested the measurement properties of the constructs (latent variables) through confirmatory factor analysis using correlation matrix. CFA was used to evaluate how well the measurement items reflect latent variables in the hypothesized structure.

Composite Reliability (CR) of latent variables and Average Variance Extracted (AVE) of Squared Multiple Correlation (SMC), is presented in Table-11. 
Table-11: Reliability and validity analytical results of container terminal supply chain performance measurement model

\begin{tabular}{|c|c|c|c|c|}
\hline $\begin{array}{l}\text { Perspectives } \\
\text { (Constructs) }\end{array}$ & Criteria & $\begin{array}{c}\text { Standardized Factor } \\
\text { Loadings }\end{array}$ & $\begin{array}{c}\text { Composite Reliability } \\
\text { (CR) }\end{array}$ & AVE \\
\hline \multirow{2}{*}{ FP } & OR & 1.00 & \multirow{2}{*}{0.8158} & \multirow{2}{*}{0.6995} \\
\hline & NI & 0.63 & & \\
\hline \multirow{3}{*}{$\mathrm{CP}$} & RPK & 1.00 & \multirow{3}{*}{0.8401} & \multirow{3}{*}{0.6477} \\
\hline & NP & 0.57 & & \\
\hline & RTK & 0.79 & & \\
\hline \multirow{2}{*}{ BP } & $\mathrm{EC}$ & 0.84 & \multirow{2}{*}{0.8505} & \multirow{2}{*}{0.7400} \\
\hline & $\mathrm{CC}$ & 0.88 & & \\
\hline \multirow{2}{*}{ LG } & LC & 0.91 & \multirow{2}{*}{0.6616} & \multirow{2}{*}{0.5199} \\
\hline & $\mathrm{OE}$ & 0.46 & & \\
\hline
\end{tabular}

Convergent validity was verified for each factor loading. Factor loadings of NP and OE are relatively poor. ROA are poor. It indicates that these measurement items are not strongly correlated its assumed theoretical construct.

Composite reliability of for each of the constructs of FP, CP, BP and LG are $0.8158,0.8401$, 0.8505 , and 0.66616 .

The average variance extracted (AVE) for each of the constructs of FP, CP, BP and LG are $0.6995,0.6477,0.7400$ and 0.5199 respectively. AVE values are more than threshold value of 0.5 indicates good convergent validity. Convergent validity is the degree of confidence we have that a latent variable is well measured by its indicators.

\subsubsection{Path Diagram}

The path diagram of the model is shown in Fig.4.

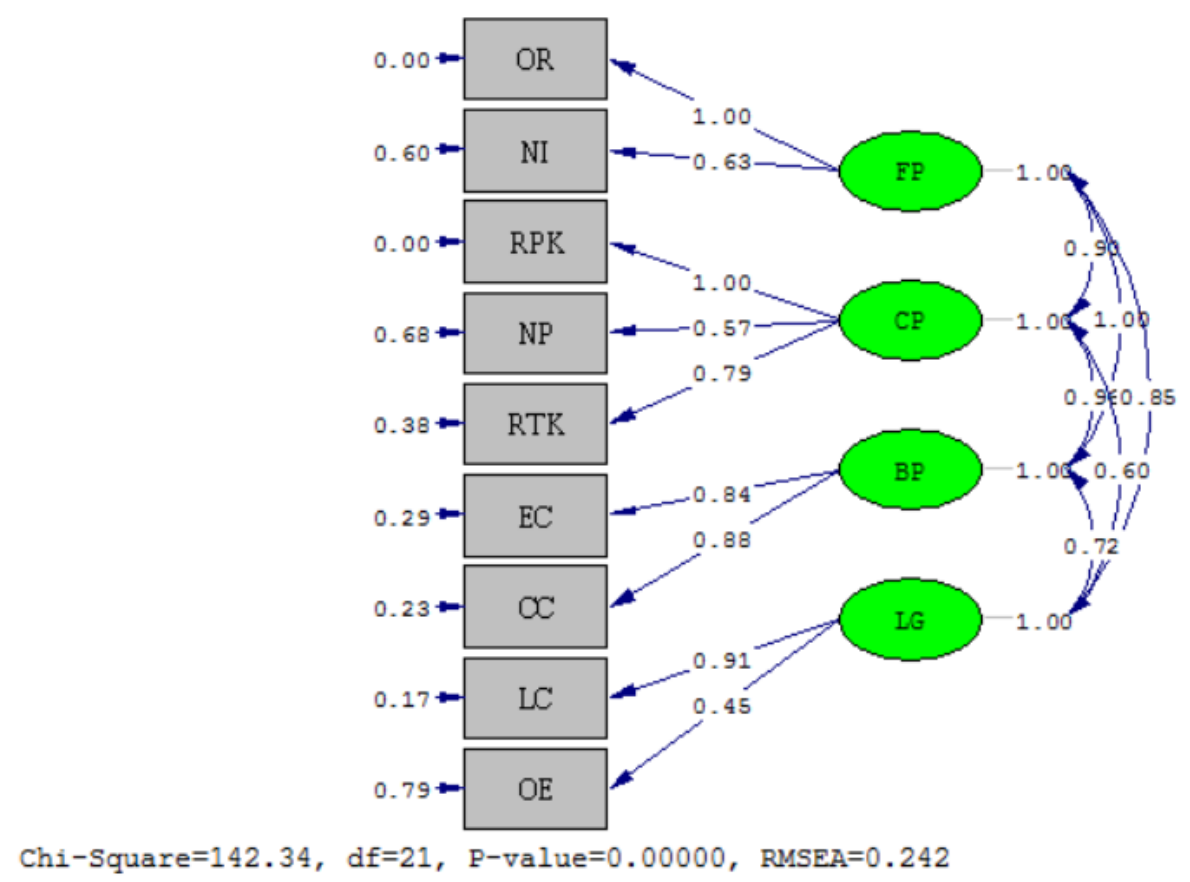

Figure 4: Path diagram

\subsubsection{Model Fit Indices}

The model fitting process involves determining the goodness-of fit between the hypothesized model and the sample data. Fit indices of the conceptual model obtained through CFA are shown in the Table-12. 
Development of Balanced Score Card Framework for Performance Evaluation of Airlines

Table-12: Fit indices of structure model

\begin{tabular}{|c|c|c|c|}
\hline Indicators & Propriety Indicators & Research Findings & Remarks \\
\hline \multirow{3}{*}{ Absolute Propriety Indicators } & $\chi^{2} /$ d.f & 6.78 & Lack of fit \\
\cline { 2 - 4 } & GFI & 0.76 & Lack of fit \\
\cline { 2 - 4 } & AGFI & 0.48 & Lack of fit \\
\cline { 2 - 4 } & SRMR & 0.13 & Poor fit \\
\cline { 2 - 4 } & RMSEA & 0.24 & Lack of fit \\
\hline \multirow{3}{*}{ Relative Propriety Indicators } & NNFI & 0.76 & Lack of fit \\
\cline { 2 - 4 } & NFI & 0.85 & Lack of fit \\
\cline { 2 - 4 } & CFI & 0.86 & Lack of fit \\
\cline { 2 - 4 }
\end{tabular}

The Chi square goodness of fit metric is obtained as 142.34. The goodness-of-fit index (GFI) of was the very first standardized fit index GFI $=0.76$ indicates poor model fit (GFI > .90). Also, AGFI, NNFI, NFI and CFI are obtained as $0.48,0.76,0.85$ and 0.86 respectively. It indicates overall poor fit of the model with the criteria under the respective construct.

In the study, RMSEA and SRMR are obtained as 0.24 and 0.13 respectively indicates the poor model fit since higher values are obtained. Overall measurement model fitness of 6.78 is obtained. It indicates that the overall measurement model fitness is poor since $\chi^{2} /$ d.f value is more than 3.0.

The confirmatory factor analysis with 4 constructs having 9 items is not valid measurement model of balanced scorecard of Airlines. In this context either NP from CP or OE from LP need to be omitted. OE cannot be omitted since the LP construct contains only two criteria. Hence, proceed for further analysis by removing NP factor from customer perspective.

\subsection{Confirmatory Factor Analysis Model-III}

The proposed model is based on four main constructs of balanced scorecard namely: (i) Financial Perspective (FP) (ii) Customer Perspective (CP) (iii) Internal Business perspective (IBP) and learning and Growth Perspective (LGP)

Based on the literature review, this research suggests the following hypotheses. The detailed discussion of hypotheses development follows.

H1: $\quad$ Operating Revenue and Net Income are the measurement items of financial perspective.

H2: $\quad$ Revenue Passenger Kilometer and Revenue Ton Kilometer are the measurement items of Customer perspective.

H3: $\quad$ Energy Cost and Capital Cost are the measurement items of Internal Business Process perspective.

H4: Labour Coat and Operating Expenses per employee are the measurement items of Learning and Growth perspective

\subsubsection{Correlation Analysis}

In this study, correlation between nine criteria under four perspectives is determined using Lisrel 8.8 to input the same for confirmatory factor analysis. The correlation matrix so obtained is presented in Table-13.

Table-13: Correlation matrix

\begin{tabular}{|c|c|c|c|c|c|c|c|c|}
\hline Criteria & OR & NI & RPK & RTK & EC & CC & LC & OE \\
\hline OR & 1 & & & & & & & \\
\hline NI & 0.635 & 1 & & & & & & \\
\hline RPK & 0.908 & 0.652 & 1 & & & & & \\
\hline
\end{tabular}




\begin{tabular}{|c|c|c|c|c|c|c|c|c|} 
RTK & 0.690 & 0.467 & 0.792 & 1 & & & & \\
\hline EC & 0.865 & 0.509 & 0.797 & 0.574 & 1 & & & \\
\hline CC & 0.864 & 0.574 & 0.866 & 0.723 & 0.737 & 1 & & \\
\hline LC & 0.765 & 0.382 & 0.526 & 0.369 & 0.613 & 0.503 & 1 & \\
\hline OE & 0.532 & 0.419 & 0.523 & 0.348 & 0.489 & 0.413 & 0.415 & 1 \\
\hline
\end{tabular}

To provide evidence for construct validity of latent variables (FP, CR, IBP and LGP) CFA model is assessed. Only valuable indicators of each latent variable would be maintained in model by utilizing factor loading.

\subsubsection{Analysis of Reliability and Validity}

The study tested the measurement properties of the constructs (latent variables) through confirmatory factor analysis using correlation matrix. CFA was used to evaluate how well the measurement items reflect latent variables in the hypothesized structure.

Composite Reliability (CR) of latent variables and Average Variance Extracted (AVE) of Squared Multiple Correlation (SMC), is presented in Table-14.

Table-14: Reliability and validity analytical results of container terminal supply chain performance measurement model

\begin{tabular}{|c|c|c|c|c|}
\hline $\begin{array}{l}\text { Perspectives } \\
\text { (Constructs) }\end{array}$ & Criteria & $\begin{array}{c}\text { Standardized Factor } \\
\text { Loadings }\end{array}$ & $\begin{array}{c}\text { Composite Reliability } \\
\text { (CR) }\end{array}$ & AVE \\
\hline \multirow{2}{*}{ FP } & OR & 1.00 & \multirow[b]{2}{*}{0.8158} & \multirow[b]{2}{*}{0.6995} \\
\hline & NI & 0.63 & & \\
\hline \multirow{2}{*}{$\mathrm{CP}$} & RPK & 1.00 & \multirow{2}{*}{0.8929} & \multirow{2}{*}{0.8089} \\
\hline & RTK & 0.78 & & \\
\hline \multirow{2}{*}{ BP } & $\mathrm{EC}$ & 0.84 & \multirow{2}{*}{0.8505} & \multirow{2}{*}{0.7400} \\
\hline & $\mathrm{CC}$ & 0.88 & & \\
\hline \multirow{2}{*}{ LG } & LC & 0.91 & \multirow{2}{*}{0.6616} & \multirow{2}{*}{0.5199} \\
\hline & $\mathrm{OE}$ & 0.46 & & \\
\hline
\end{tabular}

Convergent validity was verified for each factor loading. Factor loadings of criteria are relatively acceptable $(>0.4)$. It indicates that these measurement items are moderately correlated its assumed theoretical construct.

Composite reliability of for each of the constructs of FP, CP, BP and LG are $0.8158,0.8929$, 0.8505 , and 0.66616 indicates good reliability.

The average variance extracted (AVE) for each of the constructs of FP, CP, BP and LG are $0.6995,0.8089,0.7400$ and 0.5199 respectively. AVE values are more than threshold value of 0.5 indicates good convergent validity. Convergent validity is the degree of confidence we have that a latent variable is well measured by its indicators.

\subsubsection{Path Diagram}

The path diagram of the model is shown in Fig.5. 


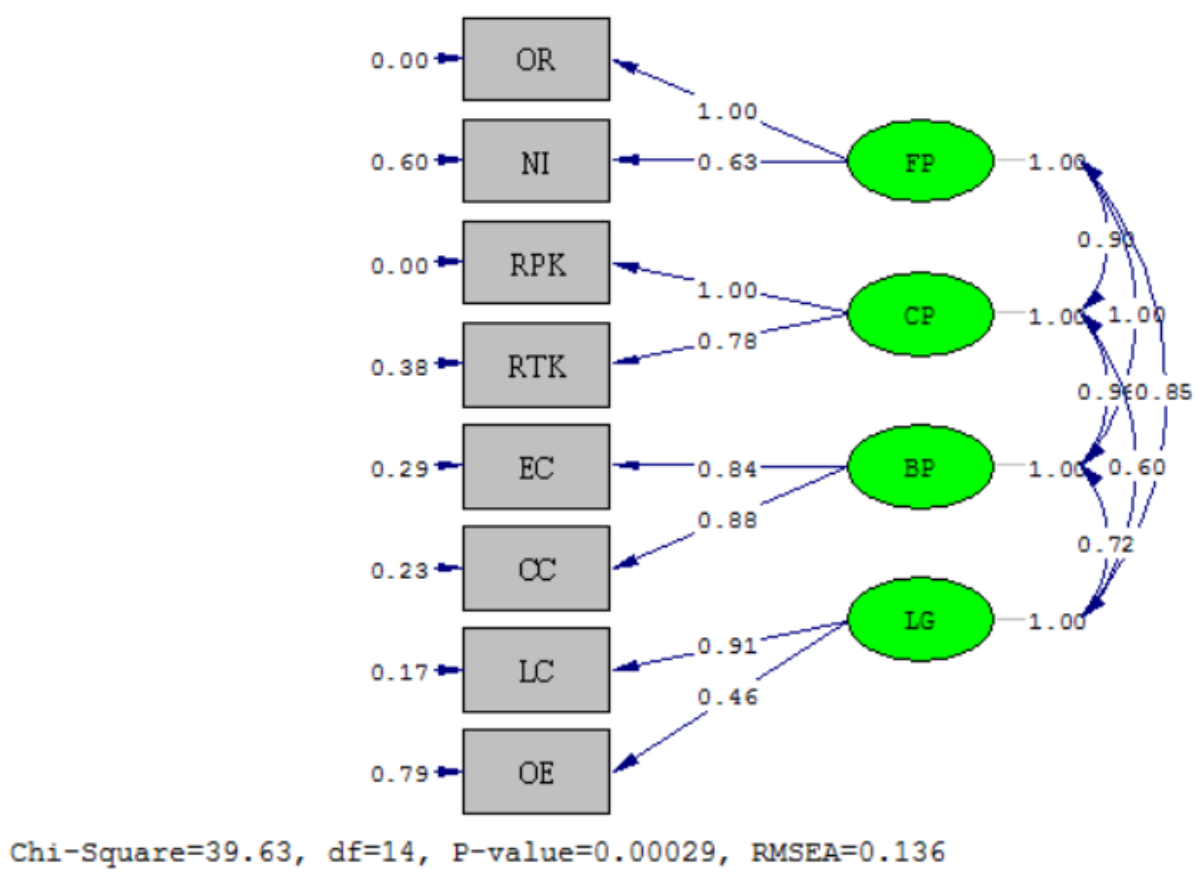

Figure 5: Path diagram

\subsubsection{Model Fit Indices}

The model fitting process involves determining the goodness-of fit between the hypothesized model and the sample data. Fit indices of the conceptual model obtained through CFA are shown in the Table-15.

Table-15: Fit indices of structure model

\begin{tabular}{|c|c|c|c|}
\hline Indicators & Propriety Indicators & Research Findings & Remarks \\
\hline \multirow{4}{*}{ Absolute Propriety Indicators } & $\chi^{2} / \mathrm{d} . \mathrm{f}$ & 2.83 & Good fit \\
\cline { 2 - 4 } & GFI & 0.91 & Good fit \\
\cline { 2 - 4 } & AGFI & 0.77 & Mediocre fit \\
\cline { 2 - 4 } & SRMR & 0.08 & Good fit \\
\cline { 2 - 4 } & RMSEA & 0.136 & Gediocre fit \\
\hline \multirow{3}{*}{ Relative Propriety Indicators } & NNFI & 0.95 & Good fit \\
\cline { 2 - 4 } & NFI & 0.95 & Good fit \\
\cline { 2 - 4 } & CFI & 0.98 & Good fit \\
\hline
\end{tabular}

The Chi square goodness of fit metric is obtained as 39.63. The goodness-of-fit index (GFI) of was the very first standardized fit index GFI $=0.91$ indicates good model fit (GFI $>0.90)$. Also, AGFI, NNFI, NFI and CFI are obtained as $0.77,0.95,0.95$ and 0.98 respectively. It indicates overall good model fit with the eight criteria under four constructs.

In the study, RMSEA and SRMR are obtained as 0.136 and 0.08 respectively indicates satisfactory model fit since lower values are obtained. Overall measurement model fitness of 2.83 is obtained. It indicates that the overall measurement model fitness is good since $\chi^{2} / \mathrm{d}$.f value is less than 3.0.

Hence In this study, the confirmatory factor analysis with 4 constructs having 8 items is a valid measurement model of balanced scorecard of Airlines.

\subsection{CRITIC Method}

In this study, relative weights of the criteria of balanced scorecard perspectives are determined through CRITIC method as per methodology. 
Table-16: Standard deviation of financial ratios

\begin{tabular}{|c|c|c|c|c|c|c|c|c|}
\hline Criteria & OR & NI & RPK & RTK & EC & CC & LC & OE \\
\hline Standard Deviation & 0.2499 & 0.1945 & 0.2282 & 0.1915 & 0.1863 & 0.2215 & 0.2026 & 0.1127 \\
\hline
\end{tabular}

From Table-16 it is observed that highest standard deviation (0.2499) is obtained with OR (Operating revenue). OE (Operating Expenses) is obtained low standard deviation (0.1127). A high standard deviation implies that, on average, data points are all pretty far from the average. A low standard deviation means most points are very close to the average.

\subsubsection{Correlation Coefficient Matrix}

Linear correlation coefficients between the financial ratios are determined.

Table-17: Correlation coefficient of criteria

\begin{tabular}{|c|c|c|c|c|c|c|c|c|}
\hline Criteria & OR & NI & RPK & RTK & EC & CC & LC & OE \\
\hline OR & 1 & 0.635 & 0.908 & 0.69 & 0.865 & 0.864 & 0.765 & 0.532 \\
\hline NI & 0.635 & 1 & 0.652 & 0.467 & 0.509 & 0.574 & 0.382 & 0.419 \\
\hline RPK & 0.908 & 0.652 & 1 & 0.792 & 0.797 & 0.866 & 0.526 & 0.523 \\
\hline RTK & 0.69 & 0.467 & 0.792 & 1 & 0.574 & 0.723 & 0.369 & 0.348 \\
\hline EC & 0.865 & 0.509 & 0.797 & 0.574 & 1 & 0.737 & 0.613 & 0.489 \\
\hline CC & 0.864 & 0.574 & 0.866 & 0.723 & 0.737 & 1 & 0.503 & 0.413 \\
\hline LC & 0.765 & 0.382 & 0.526 & 0.369 & 0.613 & 0.503 & 1 & 0.415 \\
\hline OE & 0.532 & 0.419 & 0.523 & 0.348 & 0.489 & 0.413 & 0.415 & 1 \\
\hline
\end{tabular}

Correlation coefficients are used in statistics to measure how strong a relationship is between two variables. 1 indicates a strong positive relationship. -1 indicates a strong negative relationship. A result of zero indicates no relationship at all. From table 4.10 it is observed that OR is showing high positive correlation with all other criteria. NI is showing high correlation with RPK, EC and CC. RTK is showing high correlation with EC and CC. EC is showing high correlation with EC and LC. CC is showing high correlation with EC.

\subsubsection{Measure of Conflict}

Measure of conflict is determined and shown in Table-18.

Table-18: Measure of conflict

\begin{tabular}{|c|c|c|c|c|c|c|c|c|}
\hline Criteria & OR & NI & RPK & RTK & EC & CC & LC & OE \\
\hline Measure of Conflict & 2.982 & 5.370 & 3.434 & 5.040 & 3.871 & 4.040 & 4.872 & 4.985 \\
\hline
\end{tabular}

From Table-18, it is observed that there is a high measure of conflict of 5.370 with NI and low measure of conflict of 2.982 is obtained with OR.

\subsubsection{Amount of Information in the Criteria}

The amount of information contained in the criteria is determined. The values of Amount of information in the criteria are shown in Table-19.

Table-19: Information content of criteria

\begin{tabular}{|c|c|c|c|c|c|c|c|c|}
\hline Criteria & OR & NI & RPK & RTK & EC & CC & LC & OE \\
\hline Information Content & 0.435 & 0.654 & 0.442 & 0.581 & 0.450 & 0.514 & 0.694 & 0.435 \\
\hline
\end{tabular}

From Table-19 it is observed that, LC is obtained the highest value (0.694). Hence LC transmits the largest information and it has the highest relative importance for the decisionmaking process. 


\subsubsection{Relative Weights of Criteria}

Relative weights of criteria are obtained and the relative weights of criteria are shown in Table20 .

Table-20: Relative weights of FRs

\begin{tabular}{|c|c|c|c|c|c|c|c|c|}
\hline Criteria & OR & NI & RPK & RTK & EC & CC & LC & OE \\
\hline Relative Weight & 0.1034 & 0.1555 & 0.1051 & 0.1383 & 0.1070 & 0.1222 & 0.1651 & 0.1035 \\
\hline
\end{tabular}

From Table-20 it is observed that highest weight of 0.1651 is obtained with LC and the lowest weight $(0.1034)$ is obtained with OR. The relative importance order of criteria is presented below.

Relative importance of FRs: $\mathrm{LC}>\mathrm{NI}>\mathrm{RTK}>\mathrm{CC}>\mathrm{EC}>\mathrm{RPK}>\mathrm{OE}>\mathrm{OR}$

\section{CONCLUDING REMARKS}

The present findings provide evidence to support that this is a valid instrument to determine performance dimensions of airlines based on balanced scorecard. This study suggested that four factor model with 08 items in respect of balanced score card for performance evaluation of airlines container terminals had a good fit. In this study performance measurement framework for evaluation of airlines based on balanced scorecard is developed using Integrated CFA and CRITIC methods.

This research aims to develop performance measurement instrument of Airlines based on balanced score card, which can be concluded as follows:

- The reliability test results indicate that performance measurement instrument of Airlines has good reliability, because the calculation results show that $\mathrm{CR}$ is ranging from is 0.6616 to 0.8929 and VE is ranging from 0.5199 to 0.8089 . The value is already qualified and far exceeds the critical limit that is Construct Reliability (CR) Variance extracted $(\mathrm{VE}) \geq 0.5$.

- Performance measurement instrument of Airlines consists of three dimensions, eight factors under four constructs, empirically has met the validity and reliability standards.

- The study made in this paper uses objective rating method by implementing CRITIC method. To measure the relative importance of criteria which will be useful for assessment of alternatives.

- In this study, relative importance of eight criteria under balanced scorecard perspectives is obtained as Labour Cost (0.1651), Net Income (0.1555), Revenue Ton kilometer (0.1383), Capital cost (0.1222), Energy Cost (0.1070), Revenue per passenger Kilometer (0.1051), Operating Expenses (0.1035) and Operating Revenue $(0.1034)$ is obtained.

- Similar Relative importance of Operating expenses and Operating revenue is obtained.

Results of the development of this instrument can be used as a reference in the development of similar instruments. In addition, these instruments still need to be tested again with larger criteria and sample by using various other methods and analytical techniques in order to obtain a standardized instrument. Also, the study made in this paper will be useful for performance evaluation of Airlines in specific and any other alternative business organizations in general based on balanced scorecard perspective. 


\section{REFERENCES}

[1] Kaplan, R.S and Norton, D.P., The Balanced Scorecard-Measures That Drive Performance, Harvard Business Review, 70, 1992, pp.71-79.

[2] Kaplan, R.S., Norton, D.P. Linking the Balanced Scorecard to Strategy, California Management Review. 1996, 39, pp.53-79.

[3] Wang, Y.G.; Li, Y.-M.; Jan, C.L. and Chang, K.W.,Evaluating firm performance with balanced scorecard and data envelopment analysis, WSEAS Transaction on Business and Economics, 10, 2013, pp.24-39.

[4] Gusnardi, Rina Selva Johan and Iskandar Muda, Confirmatory Factor Analysis of Dimensions of The Balanced Scorecard as a Strategy for Local Government Capacity Apparatus, International Journal of Sciences and Research, Vol.73, No.1, 2017, pp.170-175

[5] Najafi, E., Aryanegad, M., Lotfi, F., \& Ebnerasould, A, Efficiency and effectiveness rating of organization with combined DEA and BSC. Applied Mathematical Sciences, Vol.3, 2009, pp.239-265.

[6] Eilat H., Golany, B., \& Shtub, A, R\&D project evaluation: An Intergrade DEA and Balanced Scorecard approach, Omega, Vol.36, 2008, pp.895-912.

[7] Mandy M. Cheng and Kerry A. Humphreys, The Differential Improvement Effects of the Strategy Map and Scorecard Perspectives on Managers' Strategic Judgments. The Accounting Review: May 2012, Vol.87, No.3, 2012, pp.899-924.

[8] Iskandar Muda, Erlina, Idhar Yahya and Abdillah Arif Nasution, Performance Audit and Balanced Scorecard Perspective, International Journal of Civil Engineering and Technology, 9(5), 2018, pp. 1321-1333

[9] Humphreys and Trotman, The Balanced Scorecard: The Effect of Strategy Information on Performance Evaluation Judgments, Journal of Management Accounting Research American Accounting Association, Vol.23, 2011, pp.81-98

[10] Kaplan, S., Petersen, M. J., \& Samuels, J. A, An examination of the effect of positive and negative performance on the relative weighting of strategically and non-strategically linked balanced scorecard measures. Behavioral Research in Accounting, Vol.2,No2, 2012, pp.133-151.

[11] Malina and Selto, Communicating and Controlling Strategy: An Empirical Study of the Effectiveness of the Balanced Scorecard, Journal of Management Accounting Research, Vol.13, 2007, pp.1-53.

[12] Hasan Dinçer, Ümit Hacıoglu and Serhat Yüksel, Balanced scorecard based performance measurement of European airlines using a hybrid multicriteria decision making approach under the fuzzy environment, Journal of Air Transport Management, Vol.63, 2017, pp-1733.

[13] Anirut Pipatprapa, Hsiang-Hsi Huang, Ching-Hsu Huang, An Integrated Approach For Developing Environmental Performance Evaluation Of Taiwan's Food Industry, International Journal Of Scientific \& Technology Research, Vol.5, No.06, 2016, pp.301305 .

[14] Bojuwon Mustapha and Bojuwon Yusuf Bolaji, Measuring Lecturers Commitment Scales: A Second order Confirmatory Factor Analysis (CFA), International Journal of Education and Research, Vol.3, No.3, 2015, pp.505-516.

[15] Shahid Hussain, Zhu Fangwei , Ahmed Faisal Siddiqi, Zaigham Ali and Muhammad Salman Shabbir, Structural Equation Model for Evaluating Factors Affecting Quality of Social Infrastructure Projects, Sustainability, Vol.10, No.1415, 2018, pp.1-25. 
[16] S. Gopinath, Boda Sagar Kantilal, S. Sreelakshmi, Mohammed Roshan. Performance Measurement of Residential Projects in India: A Balanced Scorecard Approach. International Journal of Civil Engineering and Technology, 7(6), 2016, pp. 698-706.

[17] David Staš, Radim Lenort, Pavel Wicher and David Holman, Green Transport Balanced Scorecard Model with Analytic Network Process Support, Sustainability, Vol.7, 2015, pp.15243-15261

[18] Agnieszka Tubis and Sylwia Werbińska-Wojciechowska, Balanced Scorecard Use in Passenger Transport Companies Performing at Polish Market, Procedia Engineering, Vol.187, 2017, pp.538-547.

[19] Frederico, Guilherme F and Cavenaghi, Vagner, The Application of the Balanced Scorecard in the Operators of Freights Railroad Transportation, POMS $19^{\text {th }}$ Annual Conference La Jolla, Califórnia, U.S.A., 2008, pp.1-20.

[20] Hamed Rahimi, Jamshid Bahmaei, Payam Shojaei, Zahra Kavosi and Mohammad Khavasi , Developing a Strategy Map to Improve Public Hospitals Performance with Balanced Scorecard and DEMATEL Approach, Shiraz E-Med J., Vol.19, No.7, 2017, pp.1-12.

[21] Saif Hassan Ali Alzaabi and Hassan Al-Dhaafri, The Effect of Strategy Management and Balanced Scorecard on Organizational Performance of UAE Civil Defense, Business Management and Strategy, Vol.18, No.1, 2018, pp.84-104.

[22] Vinh V Thai, The Impact of Port Service Quality on Customer Satisfaction: The Case of Singapore, Maritime Economics \& Logistics, DOI:10.1057/mel.2015.19, 2015, pp.1-24 